from a Correspondent

THE binding of valence electrons to atoms has been investigated since the 1940 s by paramagnetic resonance techniques, and solids produce the most interesting results. If a strong $\mathrm{DC}$ magnetic field is applied to a material, the unpaired electrons precess at a given frequency, which can be detected as a resonant absorption of energy from a microwave source. Usually the sample is set in a resonant cavity and the strength of the magnetic field changed until the absorption peak is detected. The shape of the peak is usually very narrow and can thus be examined for structure.

The use of microwave energy implies, of course, that the sample is exposed to an alternating electric field at $\mathrm{MHz}$ frequencies. Recently some experimenters have found that this pulsed electrical energy can be re-emitted from certain semiconductors at a different frequency, characteristic of the material, and that the frequency can be altered by changing the magnetic field. This suggests that some kind of magnetic splitting of levels is taking place. It also suggests that, by optimizing this emission, a new way of generating intense beams of far infrared radiation with bandwidths much narrower than those obtainable by thermal sources may have been discovered. What is more, the frequency can be tuned by means of the magnetic field.

The concept first arose when Chynoweth and co-workers (Appl. Phys. Lett., 8, 157 ; 1965), performing resonance experiments on n-type indium antimonide, unexpectedly found microwave emission rather than absorption in their resonant cavity. They simply reported the fact, however, and did not explore its possibilities. Others did likewise in the following years, and some also found that this emission would take place under DC electrical and magnetic fields as well. Kobayashi, Komatsubara and Otsuka have now examined the emission phenomenon more carefully and in doing so have increased the DC magnetic fields used, so moving the emission from the microwave region to the rarely-produced far infrared region (Phys. Rev. Lett., 30, 702; 1973).

As an additional advantage of the use of high fields, the theoretical prediction of the band-splitting phenomena is simpler. As yet they have not obtained the full spectrum of the infrared radiation for a given condition-detection is limited to a few frequencies because, in the materials available as detectors, photoconductivity is excited from discrete shallow-lying impurity centres in materials such as germanium giving a narrow and unchangeable bandpass. However, it can be said that one maximum of emission is obtained in a germanium (antimony) detector centred on $0.0098 \mathrm{eV}$ (about $120 \mu \mathrm{m}$ ) when the magnetic field strength is 11 kOersteds. A pulsed field of about $15 \mathrm{~V} \mathrm{~cm}^{-1}$ for $400 \mu \mathrm{s}$ and repeated every $70 \mathrm{~ms}$ provides optimum conditions as regards lattice heating and width of spectral peaks. The widths of the various spectral peaks which appeared could be estimated roughly by measuring intensity with the detector while the magnetic field was changed. A curve of radiation intensity versus magnetic field corresponds essentially to an emission spectrum and relative wavelengths can be estimated from it. Four peaks can be distinguished at $4.2 \mathrm{~K}$ although overlap of the peaks makes accurate measurements difficult. But plotting of the assumed order number of these peaks versus the reciprocal of the field suggests quite strongly that they are produced by the splitting of the conduction band of indium antimonide into four sub-bands. Theoretical calculations show the splitting to give the correct energy values such that emission could occur in the $100 \mu \mathrm{m}$ region.

The authors thus interpret the emis- sion to be due to the production of hot electrons by the applied electric field, in the form of strong population of the $1+$ sub-bands of the conduction band (this band would be unoccupied in the unexcited state) followed by radiative transitions from this band into the ground state and the shallow donor state associated with the ground state. The weaker peaks could derive from the fall of electrons from the $2+$ and $3+$ sub-bands. This interpretation implies that the de-excitation process may never be very efficient, as hot electrons frequently lose their energy by nonradiative phonon emission. But even a few per cent of energy converted into photon emission has proved extremely useful in gallium arsenide lamps, in which it has not been found too difficult to dissipate the waste energy. Any opportunity to use such a convenient conversion method as this-namely the direct conversion of electric currents into light-can tolerate a moderate heat-dissipation problem. The prospect of a tunable solid-state source of narrow-band infrared light in a wavelength region where other sources are scarce should certainly be examined further until it is seen whether a working model is more bulky and inconvenient than that provided by other methods.

\title{
ATMOSPHERE
}

\section{Nitric Oxide from Bombs and Planes}

THE amount of nitric oxide fnjected into the upper atmosphere by the atmospheric nuclear tests of about ten years ago was about the same as the amount which is expected to be produced by a fleet of 500 supersonic transports. This calculation is given by $\mathbf{H}$. M. Foley and M. A. Ruderman of Columbia University, New York, in an article in the Journal of Geophysical Research (78, 4441 ; 1973), and clearly they are thinking that an examination of the effects of the nitric oxide from the tests will give clues as to the likely effects on the atmosphere if SSTs become widely used by the world's airlines.

The fear is of course that small amounts of nitric oxide in the atmosphere can remove large amounts of ozone by the reactions

$$
\begin{aligned}
& \mathrm{NO}+\mathrm{O}_{3} \rightarrow \mathrm{NO}_{2}+\mathrm{O}_{2} \\
& \mathrm{NO}_{2}+\mathrm{O} \rightarrow \mathrm{NO}+\mathrm{O}_{2} \\
& \mathrm{NO}_{2}+h \nu \rightarrow \mathrm{NO}+\mathrm{O}
\end{aligned}
$$

thus reducing one of the barriers to incoming radiation from the Sur. Foley and Ruderman estimate that the amount of extra nitric oxide getting into the stratosphere during a year of intense testing in the atmosphere of high yield nuclear explosions is about $10^{34}$ molecules, whereas a fleet of 500 SSTs flying seven hours per day would give, according to one calculation, about $3 \times 10^{34}$ molecules per year. The natural injection rate of nitric oxide into the stratosphere, on the other hand, also seems to be in the region of $10^{34}$ molecules per year.

So what was the effect on the atmospheric ozone of the years of intense atmospheric testing? Foley and Ruderman have considered the period from 1960 to 1964 , when worldwide monitoring of ozone was already under way. This interval includes periods in 1961 and 1962 when the Soviet Union and the United States both carried out several atmospheric tests, but there was no sign of any related decrease in the ozone content of the atmosphere. Something like a ten per cent reduction in ozone content might have been expected.

The conclusion seems to be that the ozone content of the atmosphere is not particularly sensitive to the amount of nitric oxide expected to be injected by nuclear tests or by SSTs. Why this should be so, in apparent contradiction to expectations, is not at all clear. But presumably there must be some faults in the models that are used for the behaviour of nitric oxide and ozone in the stratosphere. 